\title{
Staged Fault Test Evaluation of High-Voltage Equipment Maintenance Safety Grounding at a Large Hydro-Electric Powerplant
}

\author{
P. L. Atwater, Member, IEEE, P.E. \\ U.S. Bureau of Reclamation \\ Denver, Colorado 80225
}

\author{
J. M. DeHaan, Member, IEEE, P.E. \\ U.S. Bureau of Reclamation \\ Denver, Colorado 80225
}

\begin{abstract}
The effectiveness of temporary personal protective safety grounding for high-voltage equipment is investigated with staged fault tests conducted by the Bureau of Reclamation at the Hoover Hydro-electric Powerplant, Nevada. Several equipment work site grounding configurations were constructed with personal protective ground cables connected to a $16.5-\mathrm{kV}, 115-\mathrm{MVA}$ generator bus. The bus was faulted, causing high current to flow in the ground cables at the work site. Instrumentation recorded selected worker contact voltages and body currents during the faults. Measured ground cable fault voltage drop is examined and shown to contain both resistive and reactive voltage drop components, with the reactance component becoming significant at high current. An approach for the prediction of ground cable composite fault voltage drop, worker exposure voltage, and the dependence of both upon work site grounding geometry is provided.
\end{abstract}

Keywor $r_{i}$ protective safety grounding, high-voltage safety, staged $f_{z} \ldots$ grounding tests

\section{INTRODUCTION}

The Bureau of Reclamation (Reclamation) operates 58 hydro-electric powerplants having an installed capacity of more than 14,000 megawatts, in addition to a variety of large pumping plants having synchronous motors up to 60,000 horsepower in size. Reclamation, along with other electric utilities, strives to create a safe working environment when equipment maintenance is performed requiring direct contact with de-energized high-voltage (over 600 volts) circuits.
In this respect, high-voltage plant equipment is not considered safe for direct contact with a de-energized circuit until it is adequately grounded with temporary personal protective safety ground cables.

The concept of providing an electrically safe working environment by grounding high-voltage equipment is well recognized in the industry $[1,2,3]$, although intended largely for transmission facilities. However, safety grounding in generation/pumping facilities can be just as important and perhaps pose even a greater duty on temporary personal protective ground cables due to higher available fault current at the facility. Traditional methods for determining safety ground cable ampacity and fault voltage drop (exposure voltage) simply use Ohm's law to predict the cable resistive IR voltage drop. Ignoring cable reactance can cause the worker exposure voltage at a grounded work site to exceed the utility established limit, even though the protective ground cable has the necessary ampacity and low resistance for the application. It will be shown that the geometry of placement of safety grounds at the work site can greatly influence the worker exposure voltage during electrical faults.

The following staged fault field test data and exposure voltage calculation procedure are a result of ongoing Reclamation electrical research in safety of electric power facilities. It is intended to provide insight for the prediction and application of safe maintenance grounding of highvoltage equipment.

\section{FIELD TEST PROCEDURE}

The first test objective was to create an actual high-voltage equipment grounded work site that is typical within a large electric power facility. Personal protective safety grounds would be applied according to typical Reclamation procedure to create the staged work site. A second test objective was to subject the staged work site to substantial fault current in the safety ground cable to produce test results comparable with real world situations. Several locations around the work site would be identified where human contact with the grounded high-voltage circuit could occur. These locations would be instrumented to measure the exposure voltage during both 
single- and three-phase faulted conditions.

The Hoover Hydro-electric Powerplant, Hoover Dam, Nevada, was chosen for the staged fault test site. The powerplant contains 17 generators of various ratings and having a total output of 2,064 megawatts. Unit N8 and associated segregated-phase bus were isolated from the power system and used as the source of fault current for the staged grounded work site. At the time of these tests, the generator was rated $115 \mathrm{MVA}$ at $16.5 \mathrm{kV}$ (a scheduled uprate rewind followed these tests). The grounded work site was created at a location of isolated generator bus which allowed the closing of the generator terminal circuit breaker to apply fault current to the work site as shown in Fig. 1. Portions of metal enclosures for the $16.5-\mathrm{kV}$ bus were removed as necessary to gain access to the grounding and measurement points. Four faults were applied to this staged grounded work site as described below.

Test 1 - Single-phase grounding: Only the A-phase ground cable was connected to the overhead generator bus and to plant ground (B \& C-phase generator bus ungrounded). The generator neutral was solidly grounded to provide zero sequence current.

Test 2 - Three-phase, single-point grounding: The A, B, \& Cphase ground cables were connected to the overhead generator bus and to a common plant ground connection point (same location as for Test 1 ). The cables were mildly restrained with rope in their natural hanging positions to prevent whipping action from the forces of the fault current.

Test 3 - Three-phase, single-point (bundled) grounding: Similar to Test 2, but the vertical hanging portion of the three ground cables were tightly bundled together with large nylon ties.

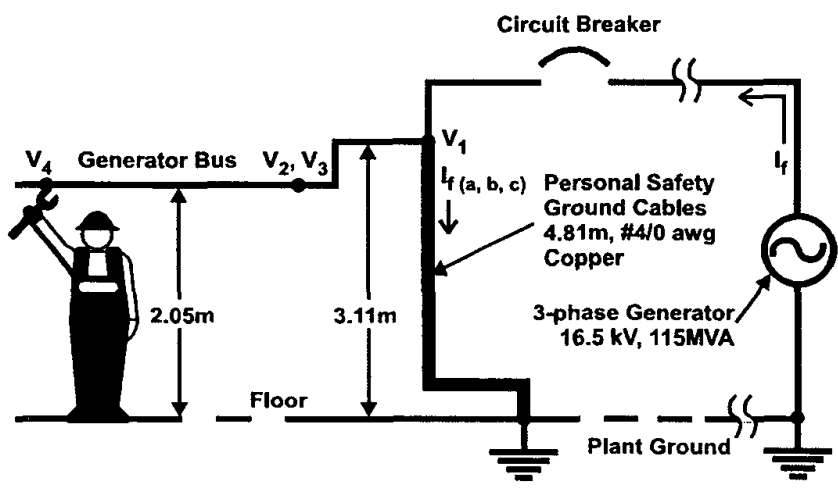

Fig. 1. Single-line representation of staged grounded work site.
Test 4 - Three-phase, Tee grounding: The overhead generator bus was bonded in a 3-phase short with two short lengths of ground cables connected directly line-to-line between the bus conductors at the ceiling (not shown in Fig. 1). The 3-phase short at the bus was connected to plant ground with only the A-phase ground cable in Fig. 1.

Instrumentation was installed to measure the protective safety ground cable fault currents, cable voltage drop, and bus contact potentials shown in Fig. 1. The $I_{f}$ ground cable currents (Ia, Ib, Ic) are self explanatory. Contact potentials $V_{l}$, $V_{2}, V_{3}$, and $V_{4}$ are described as follows:

$V_{I}$ - A-phase safety ground cable internal impedance voltage drop (voltage drop due conductor resistance plus internal reactance) with minimum additional induced voltage due to mutual inductance with the measurement circuit. This is the ideal minimum worker contact voltage if the safety ground cable could shunt directly across the body. The voltage probe lead wires were connected to the generator bus and plant ground end of the safety ground cable, and were routed alongside and taped to the surface of the cable jacket. The measurement includes the voltage drops across the cable clamps and fittings.

$V_{2}$ - Contact potential difference between the A-phase generator bus and the plant ground end of the safety ground cable. This measurement is representative of a worker simultaneously contacting the bus and another grounded object (plant equipment) located approximately 1 meter from the generator bus enclosure. Horizontal distance between the measurement points at the bus and ground end of safety cable is $2.3 \mathrm{~m}$. Linear distance along the bus between the measurement location and point of attachment of the safety ground cable to the bus is $4 \mathrm{~m}$. A closed circuit inductive ground loop comprising the safety ground cable, generator bus, plant embedded ground system, and the worker's body is formed with an area of approximately $8 \mathrm{~m}^{2}$.

$V_{3}$ - Contact potential difference between the A-phase generator bus and its grounded enclosure. This measurement represents a most probable contact between an exposed bus circuit and its immediate grounded enclosure. Same horizontal and linear distances between points as for Test 1 . Distance between contact with bus and enclosure is $0.6 \mathrm{~m}$.

$V_{4}$ - Remote contact potential difference between the Aphase generator bus and its grounded enclosure. The measurement location is approximately $45 \mathrm{~m}$ from the point of attachment of the safety ground cables to the bus. This case represents a situation where the worker is unable to place the safety grounds close to the work site due to physical restrictions. The work site is technically grounded, but a large inductive ground loop is formed with the safety ground cables, bus, and the worker: 
Table 1. Staged fault test data for safety grounded work site.

\begin{tabular}{|c|c|c|c|c|c|c|c|}
\hline Test & $I_{a}$ & $\begin{array}{l}\text { und Cable C } \\
\qquad \begin{array}{c}(\mathrm{kA}) \\
I_{b}\end{array}\end{array}$ & $\begin{array}{l}\text { rent } \\
\qquad I_{c}\end{array}$ & $\begin{array}{c}V_{l} \\
(\mathrm{~V})\end{array}$ & $\begin{array}{c}V_{2} \\
(\mathrm{~V})\end{array}$ & $\begin{array}{c}V_{3} \\
(V)\end{array}$ & $\begin{array}{c}V_{4} \\
\text { (V) }\end{array}$ \\
\hline 1 & $14.3 \angle 0$ & ---- & -- & $14 \angle 16$ & $35 \angle 60$ & $45 \angle 60$ & $46 \angle 60$ \\
\hline 2 & $11.5 \angle 0$ & $11.3 \angle-120$ & $11.6 \angle 120$ & $11.7 \angle 17$ & $22 \angle 40$ & $22.5 \angle 40$ & $25 \angle 52$ \\
\hline 3 & $10.8 \angle 0$ & $10.8<-120$ & $10.6 \angle 120$ & $11.5 \angle 15$ & $16.8 \angle 60$ & $16.6 \angle 60$ & $17.5 \angle 60$ \\
\hline 4 & $\langle .025$ & [10.8 øab] & {$[12.7 \oslash b c]$} & $\langle 1$ & $<2$ & $\langle 1$ & $\langle 1$ \\
\hline
\end{tabular}

Measurement instrumentation consisted of specialized current transformer and voltage divider probes installed at the respective measurement points. The current transformers were of nonmagnetic core Rogowski coil devices to avoid problems of saturation. The voltage dividers provided a 500 ohm load at the measurement point to simulate the human body core resistance, thus permitting realistic calculation of body current based on the measured voltage. ${ }^{1}$ The low level electrical signals from these devices were transmitted at the points of measurement via a fiber-optic system to remote digital storage chart recording equipment. The electrical configuration of the instrumentation avoided unintentional ground loops or other stray pickup which could have introduced noise into the measurements. ${ }^{2}$ Approximately nine cycles of $60 \mathrm{~Hz}$ data (fault clearing time) were recorded for each test.

\section{FIELD TEST RESULTS}

The recorded fault test data are summarized in Table $1 .{ }^{3}$ The generator prefault terminal voltage was adjusted to below rated voltage to limit the fault current and mechanical stress imposed on the generator. Higher current could have been obtained, but was not necessary for these tests. All measured voltages and currents are shown as rms, phasor values with the zero reference angle assigned to the A-phase ground cable current. Positive angle leads the reference.

\footnotetext{
${ }^{1}$ Reclamation personal protective grounding safety procedure conservatively uses 500-ohm core body resistance in determining maximum allowable exposure contact voltage. Industry standards vary [4].

${ }^{2}$ Noise immunity was demonstrated with one instrumentation record of a dummy voltage divider probe having input shorted (no signal input) and connected to plant ground. No output was recorded during a fault test.

${ }^{3}$ Values in table are approximate in some cases due to moderate harmonic waveform distortion.
}

Several observations can be made about the data in Table 1. It is clearly evident that for a given fault current in the safety ground cable the worker contact (exposure) voltage increases as the measurement point on the bus moves away from the attachment of the ground cables. The rising voltage contains a substantial inductive reactance component evidenced by the increasing leading phase angle. This demonstrates mutual coupling exists between the current-carrying safety ground cable and the closed-circuit ground loop with the worker. Note also that all the single-phase (Test 1) measured voltages, except $V_{l}$, are substantially higher in proportion to the fault current than the corresponding 3-phase test voltages. The single-phase current condition maximizes the effect of coupling due to lack of current cancellation from the other phases. This being the case, the highest exposure voltage should occur during a single-phase fault involving a larger ground loop area. The bundled conductor (Test 3) and Tee connection (Test 4) test results show an opposite effect of decreasing exposure voltage. The close physical spacing of the cables in Test 3 causes greater cancellation of the three phase fluxes in the ground loop. The Tee connection of ground cables essentially eliminates fault current in the full length cable (floor-to-ceiling) which is the predominant coupling mode into the ground loop. The measured exposure voltages are remarkably reduced and clearly demonstrate the advantage of this grounding method.

\section{TEST DATA EVALUATION}

The staged grounded work site can be modeled to determine exposure voltage in terms of magnetic coupling between the safety ground cable and a conductive ground loop which includes the worker. The approach taken here is to apply Faraday's law of induction to a conductive loop encircling a magnetic flux in air as shown in Fig. 2. 


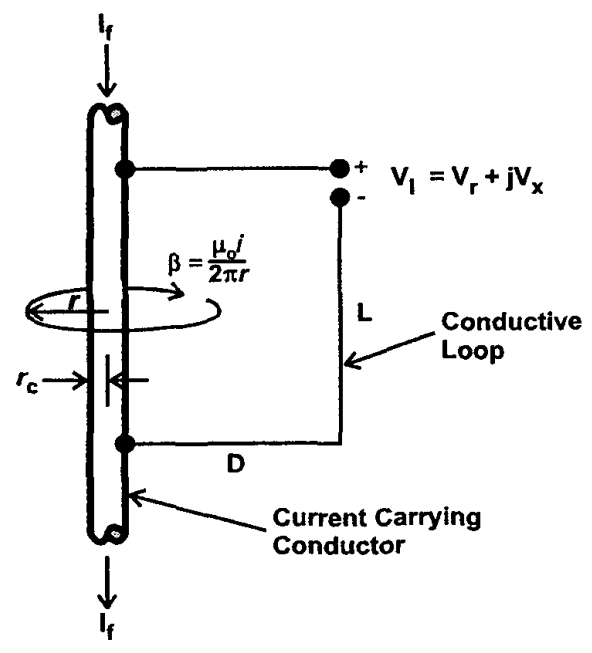

Fig. 2. Inductive ground loop principle.

The current-carrying conductor represents the safety ground cable which carries alternating fault current $I_{f}$. No current is present in the other parts of the loop. Magnetic flux encircling the conductor penetrates normal to the plane of the loop, inducing voltage $V_{x}$ in the loop. ${ }^{4}$ If the loop is rectangular with dimensions $D$ and $L$, the resulting approximate induction voltage equation is:

$$
V_{x}=j 2 \pi f I_{f} L\left[2 \ln \left(D / r_{c}\right)+0.5\right] \times 10^{-7}
$$

with units of $V_{x}$ volts, $I_{f}$ amperes, power frequency $f \mathrm{~Hz}, L$ meters, and $D$ and $r_{c}$ of same units. The $j$ operator indicates $V_{x}$ leads cable current $I_{f}$ by 90 degrees. The first term inside the bracket represents the voltage induced in the loop due to the external flux (in air) of the current-carrying conductor. The second term accounts for induced voltage from flux internal to the conductor and is independent of the size (radius) of the conductor. Resistance voltage drop $V r$ in the current-carrying conductor must be vectorially added to the induction voltage to arrive at the total voltage $V_{l}$ developed in the loop, or:

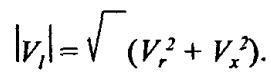

The staged grounded work site can be suitably represented with the circuit of Fig. 2 by substituting the safety ground cable for the left side (current-carrying conductor), generator bus for the top, worker's body for the right side, and plant grounding system for the bottom of the loop. Fig. 3 shows a more detailed layout of the grounded work site depicted in Fig. 1. Some portions of circuits which do not contribute to

4 In practice a closed circuit exists with the human body, causing a small current in the loop which is orders of magnitude less than $I_{f}$. This loop or body current is insignificant and the measured (loaded) loop voltage is essentially the same as the calculated open-circuit voltage. the loop voltage have been eliminated for clarity. Note that three distinct rectangular loops are required to represent the complex geometry of the work site. Exposure voltages $V_{11}$ and $V_{2 l}$ for single-phase Test 1 can be calculated realizing only the A-phase safety ground cable contributes flux in the ground loops. First, the measured resistance of the A-phase safety ground cable (including fittings and clamps) is 0.95 milliohm. The resistive voltage drop component of the ground cable is then $V_{r}=14,300 \times 0.95 \times 10^{-3}=13.6$ volts. This agrees closely with the real part of measured $V_{I}=14 \cos 16^{\circ}=13.5$ volts. Second, the reactive voltage drop component of $V_{1}$ is calculated from (1) with loop dimension $D=10.9 \mathrm{~mm}$ (test lead wire in contact with cable jacket), $r_{c}=7.8 \mathrm{~mm}$ (measured $\# 4 / 0$ awg wire radius), and $L=4.81 \mathrm{~m}$ (length of safety ground cable): $\begin{aligned} V_{l x} & =j 2 \pi 60 \times 14,300 \times 4.81[2 \ln (10.9 / 7.8)+0.5] \times 10^{-7} \\ & =j 3 \mathrm{~V} .\end{aligned}$

The total voltage drop across the cable is found by combining $V_{r}$ and $V_{I x}$ in (2) and is $V_{l l}=13.9$ volts. This agrees quite well with the measured $V_{1}$ in Table 1 . The total voltage drop is only slightly greater than the resistive drop and is not a safety concern other than to demonstrate the calculation.

Exposure voltage $V_{2 l}$ of Test 1 can be calculated in similar fashion using the dimensions of the three loop areas shown in Fig. 3. Each of the three loop reactive voltages are calculated separately from (1) and then added. This is possible because the flux penetrating one loop from the ground cable does not influence the other two. Note that $2 \pi f$ has been replaced by 377 in each computation.

$$
\begin{aligned}
V_{2 \times l} & =j 377 \times 14,300 \times 1.06[2 \ln (2.29 / 0.0078)+0.5] \times 10^{-7} \\
& =j 6.8 \mathrm{~V} \\
V_{2 \times 2} & =j 377 \times 14,300 \times 2.05[2 \ln (1.55 / 0.0078)+0.5] \times 10^{-7} \\
& =j 12.2 \mathrm{~V} \\
V_{2 \times 3} & =j 377 \times 14,300 \times 1.7[2 \ln (1.55 / 0.0078)+0.5] \times 10^{-7} \\
& =j 10.2 \mathrm{~V}
\end{aligned}
$$

The resistive voltage drop component of the loop voltage is the same as before, therefore the loop total exposure voltage is found from (2) as

$V_{2 l}=\sqrt{\left[13.6^{2}+(6.8+12.2+10.2)^{2}\right]=32.2 \mathrm{~V}}$

and is in reasonable agreement with measured $V$, in Table 1 . Note that the exposure voltage more than doubles when the point of contact with the bus moves from measurement location $V_{1}$ to $V_{2}$. 


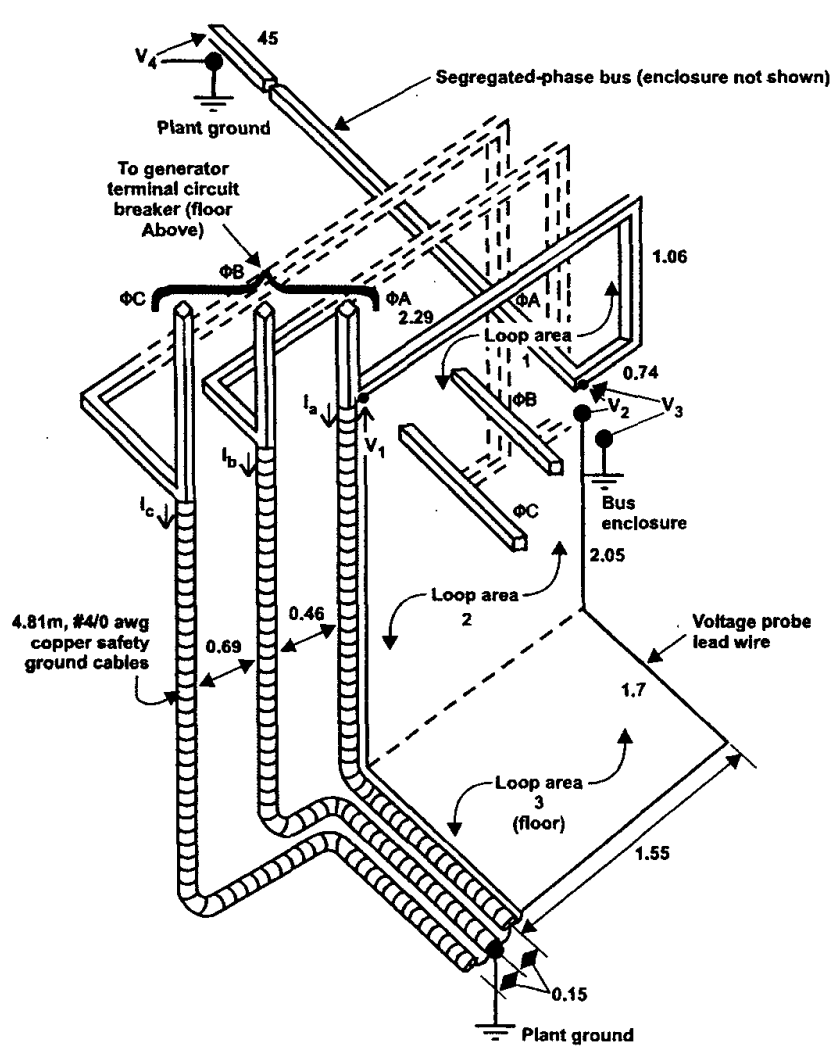

Fig. 3. Detail of grounded work site configuration.

The single-phase example above can be readily expanded for 3-phase analysis of Test 2 by accounting for the flux from the other two current-carrying safety ground cables in Fig 3 . The difference here is that only the B and C-phase flux which penetrates the closed loop defined by the A-phase cable contributes to the exposure voltages (closed-loop circuit is with A-phase only). The exposure voltages are determined by calculating the A-phase voltage component similar as for Test 1 and then adding the $\mathrm{B}$ and $\mathrm{C}$-phase component voltages induced into the loop. Calculation of $V_{\|}$is of minor concern since it should change little due to the small loop area involved. Exposure voltage $V_{2 l}$ for Test 2 is calculated as follows.

\section{Phase A}

$$
\begin{aligned}
V_{2 \times 1} & =j 377 \times 11,500 \angle 0 \times 1.06[2 \ln (2.29 / 0.0078)+0.5] \times 10^{-7} \\
& =j 5.5 \angle 0 \mathrm{~V} \\
V_{2 \times 2} & =j 377 \times 11,500 \angle 0 \times 2.05[2 \ln (1.55 / 0.0078)+0.5] \times 10^{-7} \\
& =j 9.9 \angle 0 \mathrm{~V} \\
V_{2 \times 3} & =j 377 \times 11,500 \angle 0 \times 1.7[2 \ln (1.55 / 0.0078)+0.5] \times 10^{-7} \\
& =j 8.2 \angle 0 \mathrm{~V}
\end{aligned}
$$

Phase B

$$
\begin{aligned}
V_{2 \times l} & =j 377 \times 11,300 \angle-120 \times 1.06 \times 2 \ln [(2.29+.46) / 46] \times 10^{-7} \\
& =j 1.6 \angle-120 \mathrm{~V} \\
V_{2 \times 2} & =j 377 \times 11,300 \angle-120 \times 2.05 \times 2 \ln [(1.55+.46) / .46] \times 10^{-7} \\
& =j 2.6 \angle-120 \mathrm{~V} \\
V_{2 \times 3} & =j 377 \times 11,300 \angle-120 \times 1.7 \times 2 \ln [(1.55+.15) / .15] \times 10^{-7} \\
& =j 3.5 \angle-120 \mathrm{~V}
\end{aligned}
$$

Phase C

$$
\begin{aligned}
V_{2 \times 1}= & j 377 \times 11,600 \angle 120 \times 1.06 \times 2 \ln [(2.29+.46+.69) / 1.15] \\
& \times 10^{-7} \\
= & j 1.0 \angle 120 \mathrm{~V} \\
V_{2 \times 2}= & j 377 \times 11,600 \angle 120 \times 2.05 \times 2 \ln [(1.55+.46+.69) / 1.15] \\
\times & 10^{-7} \\
= & j 1.5 \angle 120 \mathrm{~V} \\
V_{2 \times 3}= & j 377 \times 11,600 \angle 120 \times 1.7 \times 2 \ln [(1.55+.15+.15) / .3] \\
\times & \times 10^{-7} \\
= & j 2.7 \angle 120 \mathrm{~V}
\end{aligned}
$$

Note that the distance between the A to B and A to C-phase cables have been substituted for $r_{c}$ in the above $\mathrm{B}$ and $\mathrm{C}$-phase calculations, respectively. Summing the above nine values, accounting for phase displacements, gives the loop total reactive voltage $V_{2 x}=j 17.2 \angle-7$. The A-phase safety ground cable resistive voltage drop $V_{r}=11,500 \times 0.95 \times 10^{-3}=10.9 \mathrm{~V}$, therefore the loop total exposure voltage $V_{2 l}$ from (2) is approximately

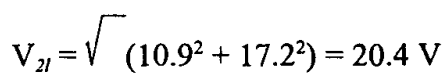

where the small error in neglecting the 7 degrees angle of $V_{2 x}$ has been ignored. This is in very good agreement with measured $V_{2}$ for Test 2 in Table 1.

The authors did not attempt to verify through calculation the measured values for $V_{3}$ and $V_{4}$ in Table 1 . The geometry of these measurements is less well defined due to the ground return paths of the generator bus enclosure and plant grounding system. However, these measurements support the general finding of increasing exposure voltage as one moves away from the point of attachment of the safety ground cables to the bus.

\section{CONCLUSION}

The test results show that the worker achieves the greatest degree of protection when hands-on contact with the deenergized equipment is as close as practical to the point of attachment of the safety ground cables. Factors such as 
physical access to the equipment and protection from violent movement of the ground cables during a fault may limit their proximity to the work site. The measured voltages from these tests are all well within Reclamation safe limits. However, the dramatic voltage rise observed with increasing working distance from the safety grounds demonstrates this may not always be the case. Some work sites having high available fault current and/or large conductive loops formed with the cable and worker may not meet the utility safe exposure voltage criteria. Tighter bundling of the safety grounds will somewhat reduce the 3-phase fault exposure voltage (Test 3) but at the risk of violent separation of cable during a fault.

The 3-phase Tee grounding (Test 4) produced the lowest fault exposure voltages. This configuration greatly reduced fault current $I_{a}$ in the single, full-length safety ground cable connected from the bus to plant ground. As a result, all the exposure voltages practically vanished. The 3-phase Tee connection may be the preferred method of grounding in many applications.

It has been shown how the actual fault exposure voltage appearing on a safety grounded generator bus can be predicted knowing the geometry of the work site. Using this method, the effectiveness of grounding for a variety of situations can be evaluated in terms of available fault current, safety ground cable size and length, and site geometry. For ease of predicting exposure voltage for 3-phase grounding, only the single-phase calculation demonstrated for Test 1 may be performed. This will result in a conservative high value, but can be further refined if necessary.

\section{ACKNOWLEDGMENT}

The authors thank the staff at Hoover Dam for their offer of the facility for testing and for their participation in coordinating and performing these tests.

\section{REFERENCES}

[1] Occupational Safety and Health Act, CFR1910.269.

[2] IEEE Std. 1048-1990, IEEE Guide for Protective Grounding of Power Lines.

[3] American Society For Testing And Materials, ASTM F855-90, Standard Specifications to Be Used on De-energized Electric Power Lines and Equipment.

[4] ANSI/IEEE Std. 80-1986, IEEE Guide for Safety in AC Substation Grounding.

\section{BIOGRAPHIES}

Philip L. Atwater received a B.S. degree in electrical engineering from Michigan State University in 1975 and then moved to Colorado to work for the Bureau of Reclamation. Phil has 24 years experience performing design, research, and field testing in areas of power and pumping plant control and protective relaying, large rotating machine diagnostics, station grounding, power system staged fault testing and special instrumentation development. He is currently Team Leader of the Electric Power and Diagnostics Team, Hydroelectric Research and Technical Services Group, and is a registered Professional Engineer in the State of Colorado. Early in his career Phil became involved with designs of equipment grounding for large power facilities and then took interest and focused on high-voltage equipment maintenance safety grounding procedure. Phil has performed several research related staged fault grounding tests on high-voltage transmission lines and in power generation facilities. He presently coordinates the Reclamation safety grounding program and provides grounding clinics and consultation.

James M. DeHaan is an electrical engineer for the Hydroelectric Research and Technical Services Group at the Bureau of Reclamation. His responsibilities include research and field testing in the areas of large rotating machine diagnostics, power quality, power system staged fault testing, machine condition monitoring, and specialized power system instrumentation development. DeHaan has a B.S. degree in electrical engineering from Dordt College and a M.S. degree in electric power from Iowa State University. He is a registered professional engineer and a member of the IEEE. 